\title{
Solução da Equação de Transporte Dependente do Tempo pela Combinação dos Métodos de Diferenças Finitas e LTAN
}

J.V.P. de OLIVEIRA ${ }^{1}$, Departamento de Matemática, Universidade Federal de Santa Maria, Av. Roraima, s/n, prédio 13, 97105-900 Santa Maria, RS, Brasil

A.V. CARDONA 2 , Faculdade de Matemática, Pontifícia Universidade Católica, Av. Ipiranga, 6681, prédio 15, 90619-900 Porto Alegre, RS, Brasil.

\begin{abstract}
Resumo. Neste trabalho apresenta-se uma nova abordagem para solução de problemas lineares de transporte dependentes do tempo, através da combinação do método das diferenças finitas e do método LTAN . Para tanto, aproxima-se a derivada temporal do fluxo angular por Diferenças Finitas Descendentes, transformandose o problema transiente resultante em um conjunto de problemas de transporte unidimensionais, os quais são então resolvidos, recursivamente, pela aplicação do método LTA $_{N}$ em cada um destes problemas, separadamente. Basicamente, o método LTA $_{N}$ consiste na resolução das equações $A_{N}$ pela técnica da transformada de Laplace na variável espacial, com inversão analítica. As equações $\mathrm{A}_{\mathrm{N}}$ são obtidas decompondo-se o fluxo angular nas direções positivas e negativas, respectivamente, como soma e subtração de duas funções definidas apenas nas direções positivas, aplicando-se então a idéia das ordenadas discretas. Simulações numéricas são apresentadas.
\end{abstract}

Palavras-chave: Problema transiente de transporte, Diferenças finitas, Método LTA $_{N}$

\section{Introdução}

Problema de Transporte é o termo comumente usado para a descrição matemática do transporte de partículas microscópicas, tais como, nêutrons, fótons e elétrons através da matéria. A equação de transporte [6], introduzida em 1872 por Boltzmann, é, na sua forma mais geral, uma equação íntegro-diferencial não-linear. Entretanto, o transporte de partículas não carregadas, tais como fótons e nêutrons, pode ser descrito pela forma linear da equação de Boltzmann. Na busca de uma solução para a equação linear unidimensional de Boltzmann, aproxima-se o seu termo integral por uma quadratura de Gauss-Legendre na variável angular e calculase a equação resultante nas direções discretas, obtendo-se assim, um sistema de

\footnotetext{
${ }^{1}$ josepol@smail.ufsm.br

2 acardona@pucrs.br
} 
equações diferenciais ordinárias, que são conhecidas como Equações de Ordenadas Discretas ou Equações $\mathrm{S}_{\mathrm{N}}[6]$.

$\mathrm{Na}$ literatura, poucos trabalhos têm objetivado o desenvolvimento de códigos computacionais para a solução de problemas de ordenadas discretas dependentes do tempo, entre os quais, podem-se citar os métodos DDIT e ACN [1]. O método DDIT consiste em usar o método de diferenças finitas denominado diamond difference na variável espacial e o método trapezoidal implícito na variável temporal das equações $\mathrm{S}_{\mathrm{N}}$. O método ACN é um método nodal que preserva a solução geral analítica das equações nodais $\mathrm{S}_{\mathrm{N}}$ integradas transversalmente com aproximações para os termos de diferença avançada, os quais são aproximados por constantes.

Por outro lado, o método $\operatorname{LTS}_{\mathrm{N}}$ [14], que resolve analiticamente as equações $\mathrm{S}_{\mathrm{N}}$ pelo uso da técnica da transformada de Laplace na variável espacial, tem sido utilizado com sucesso na resolução de problemas transientes de transporte quando combinado com o método espectral [5]. A formulação obtida através deste procedimento tem sido aplicada em problemas de transporte em domínios limitados, tanto lineares $[8,10]$, quanto não-lineares $[9,11]$ e, em problemas transientes de transporte em domínios ilimitados [12]. O método LTS $_{N}$ também foi combinado com o métodos das diferenças finitas para resolver um problema transiente de transporte unidimensional [13]. Recentemente, desenvolveu-se o método $\operatorname{LTA}_{\mathrm{N}}[2,3]$ e observou-se que este apresenta uma aparente maior taxa de convergência que o método LTS $_{\mathrm{N}}$ na solução de problemas estacionários lineares unidimencionais de transporte, implicando num menor esforço computacional. O método LTA $_{N}$, basicamente, consiste na aplicação da transformada de Laplace na variável espacial do conjunto das equações diferenciais $\mathrm{A}_{\mathrm{N}}[2]$, solução do sistema linear resultante para o fluxo angular transformado e inversão analítica do mesmo. As equações $\mathrm{A}_{\mathrm{N}}$ são obtidas decompondo-se o fluxo angular nas direções positivas e negativas, respectivamente, como soma e subtração de duas funções definidas apenas nas direções positivas, aplicando-se a idéia das ordendas discretas. Desta forma, é de se esperar que a troca da formulação $\operatorname{LTS}_{\mathrm{N}}$ pela formulação $\mathrm{LTA}_{\mathrm{N}}$ deverá resultar num ganho razoável no tempo de processamento.

Seguindo nesta direção, propõe-se neste trabalho uma nova formulação para resolver problemas transientes de transporte, combinando agora, o método das diferenças finitas com o método LTA $\mathrm{L}_{\mathrm{N}}$. Para tanto, aproximando-se a derivada temporal do fluxo angular por Diferenças Finitas Descendentes, transforma-se o problema transiente de transporte em um conjunto de problemas unidimensionais, os quais são resolvidos, recursivamente, através do método $\operatorname{LTA}_{\mathrm{N}}[2,3]$.

\section{Descrição do Problema}

Para apresentar o método proposto, considera-se o seguinte problema transiente de transporte em geometria plana, com espalhamento isotrópico, descrito pela equação:

$$
\frac{1}{v} \frac{\partial \Psi}{\partial t}(x, \mu, t)+\mu \frac{\partial \Psi}{\partial x}(x, \mu, t)+\sigma_{t} \Psi(x, \mu, t)=\frac{\sigma_{s}}{2} \int_{-1}^{1} \Psi\left(x, \mu^{\prime}, t\right) d \mu^{\prime}
$$


pelas condições de contorno,

$$
\Psi(0, \mu, t)=\Psi(0,-\mu, t), \text { se } \mu>0,
$$

e

$$
\Psi(H, \mu, t)=0, \text { se } \mu<0,
$$

e pela condição inicial,

$$
\Psi(x, \mu, 0)=\Phi(x, \mu),
$$

onde $\Psi(x, \mu, t)$ denota o fluxo angular de nêutrons na posição $x \in[0, H]$, na direção $\mu \in[-1,1]$ e no instante $t, v$ é a velocidade das partículas, $\sigma_{t}$ e $\sigma_{s}$ são definidos, repectivamente, como a seção de choque macroscópica total e de espalhamento. $\Phi(x, \mu)$ denota a condição inicial prescrita.

\section{A Aproximação no Tempo}

Visando resolver o problema (2.1-2.4), considera-se a discretização temporal $t_{m}=$ $m . \Delta t$, sendo $\Psi^{m}(x, \mu)=\Psi\left(x, \mu, t_{m}\right)$ e aproxima-se a derivada temporal na equação (2.1) por diferenças finitas atrasadas, ou seja,

$$
\frac{\partial \Psi^{m}}{\partial t}(x, \mu) \approx \frac{\Psi^{m}(x, \mu)-\Psi^{m-1}(x, \mu)}{\Delta t} .
$$

Assim, conclui-se que os fluxos angulares aproximados $\Psi^{m}(x, \mu)$ devem satisfazer um conjunto de problemas de transporte descritos pelas equações íntegrodiferenciais:

$$
\mu \frac{\partial \Psi^{m}}{\partial x}(x, \mu)+\sigma_{t}^{*} \Psi^{m}(x, \mu)=\frac{\sigma_{s}}{2} \int_{-1}^{1} \Psi^{m}\left(x, \mu^{\prime}\right) d \mu^{\prime}+Q^{m}(x, \mu),
$$

e pelas condições de contorno

$$
\Psi^{m}(0, \mu)=\Psi^{m}(0,-\mu), \text { se } \mu>0,
$$

e

$$
\Psi^{m}(H, \mu)=0, \text { se } \mu<0
$$

onde

$$
\sigma_{t}^{*}=\sigma_{t}+\frac{1}{v \cdot \Delta t}
$$

e

$$
Q^{m}(x, \mu)=\frac{\Psi^{m-1}(x, \mu)}{v \cdot \Delta t}
$$

com $m=1,2,3, \ldots$. Finalmente, uma vez determinados os fluxos $\Psi^{k}(x, \mu)$, com $k=0,1, \ldots,(m-1)$, obtém-se o fluxo $\Psi^{m}(x, \mu)$ através da resolução do problema descrito pelas equações (3.2-3.4). Cabe ressaltar, que este problema tem a mesma forma dos problemas estacionários de transporte já resolvidos, com sucesso, pelos métodos $\operatorname{LTS}_{\mathrm{N}}[14]$ e $\operatorname{LTA}_{\mathrm{N}}[3]$.

Na próxima seção, apresenta-se a solução do problema dado pelas equações (3.23.4) através do método $\mathrm{LTA}_{\mathrm{N}}$. 


\section{O Método LTA $_{\mathrm{N}}$}

Aplicando a transformação de Kuznetsov [7], para o fluxo angular

$$
\Psi^{m}(x, \mu)= \begin{cases}u^{m}(x, \mu)+v^{m}(x, \mu), & \text { se } \quad \mu>0 \\ u^{m}(x,-\mu)-v^{m}(x,-\mu), & \text { se } \quad \mu<0\end{cases}
$$

e o termo de fonte

$$
Q^{m}(x, \mu)= \begin{cases}S^{m}(x, \mu)+F^{m}(x, \mu), & \text { se } \quad \mu>0 \\ S^{m}(x,-\mu)-F^{m}(x,-\mu), & \text { se } \quad \mu<0\end{cases}
$$

substituindo essas expressões na equação (3.2), após algumas manipulações algébricas, obtém-se o conjunto de duas equações:

$$
\mu \frac{\partial}{\partial x} v^{m}(x, \mu)+\sigma_{t}^{*} u^{m}(x, \mu)=\sigma_{s} \int_{0}^{1} u^{m}\left(x, \mu^{\prime}\right) d \mu^{\prime}+S^{m}(x, \mu), \mu>0
$$

$$
\mu \frac{\partial}{\partial x} u^{m}(x, \mu)+\sigma_{t}^{*} v^{m}(x, \mu)=F^{m}(x, \mu), \mu>0 .
$$

Seguindo a idéia da aproximação $\mathrm{S}_{\mathrm{N}}[6]$ nas equaçãos (4.3-4.4), obtém-se o seguinte sistema de equações diferenciais lineares de primeira ordem

$$
\mu_{n} \frac{d}{d x} v^{m}\left(x, \mu_{n}\right)+\sigma_{t}^{*} u^{m}\left(x, \mu_{n}\right)=\sigma_{s} \sum_{k=1}^{N} \omega_{k} u^{m}\left(x, \mu_{k}\right)+S^{m}\left(x, \mu_{n}\right)
$$

$$
\mu_{n} \frac{d}{d x} u^{m}\left(x, \mu_{n}\right)+\sigma_{t}^{*} v^{m}\left(x, \mu_{n}\right)=F^{m}\left(x, \mu_{n}\right)
$$

para $n=1: N$, as quais são conhecidas como equações $A_{N}$ [4]. Aqui, $\mu_{k}$ e $\omega_{k}$ para $k=1: N$, denotam respectivamente as abscissas e pesos da fórmula de quadratura de Gauss-Legendre no intervalo $[0,1]$. Então, aplicando-se a transformada de Laplace nas equações (4.5-4.6), resulta na seguinte equação matricial

$$
(s I-A)\left[\begin{array}{c}
\bar{u}^{m}(s) \\
\bar{v}^{m}(s)
\end{array}\right]=\left[\begin{array}{c}
u^{m}(0) \\
v^{m}(0)
\end{array}\right]+\left[\begin{array}{c}
\bar{F}^{m}(s) \\
\bar{S}^{m}(s)
\end{array}\right]
$$

onde os vetores $u^{m}(x), v^{m}(x), F^{m}(x)$ e $S^{m}(x)$ são respectivamente defnidos como

$$
\left[u^{m}\left(x, \mu_{1}\right) \ldots u^{m}\left(x, \mu_{N}\right)\right]^{T},\left[v^{m}\left(x, \mu_{1}\right) \ldots v^{m}\left(x, \mu_{N}\right)\right]^{T},\left[\frac{F^{m}\left(x, \mu_{1}\right)}{\mu_{1}} \ldots \frac{F^{m}(x, \mu N)}{\mu_{N}}\right]^{T}
$$

e

$$
\left[\frac{S^{m}\left(x, \mu_{1}\right)}{\mu_{1}} \ldots \frac{S^{m}\left(x, \mu_{N}\right)}{\mu_{N}}\right]^{T}
$$


A barra denota a transformada de Laplace destes vetores. Aqui, $I$ é a matriz identidade $(2 N) \times(2 N)$ e os coeficientes $a_{i, j}$ da matriz $A(2 N) \times(2 N)$ são descritos como

$$
a_{i, j}= \begin{cases}-\frac{\sigma_{t}^{*}}{\mu_{i}}, & \text { se } i=j-N \\ -\frac{\sigma_{t}^{*}}{\mu_{j}}+\frac{\omega_{j} \sigma_{s}}{\mu_{j}}, & \text { se } i-N=j \\ \frac{\omega_{j} \sigma_{s}}{\mu_{i-N}}, & \text { se } \quad j \leq N, i>N \text { e } i-N \neq j \\ 0, & \text { em outro caso }\end{cases}
$$

A equação matricial (4.7) e sua transformada inversa de Laplace são obtidas analiticamente, usando a diagonalização da matriz $A=X D X^{-1}$ [14], resultando que

$$
\begin{aligned}
& u^{m}\left(x, \mu_{k}\right)=\sum_{i=1}^{N}\left\{\alpha(k, i) e^{d(i)(x-H)} \Gamma_{i}^{m}+\alpha(k, i+N) e^{d(i+N) x} \Gamma_{i+N}^{m}\right. \\
& +\sum_{j=1}^{N}\left[\alpha(k, i) \int_{H}^{x} e^{d(i)(x-\tau)}\left\langle\alpha^{-1}(i, j) F^{m}\left(\tau, \mu_{j}\right)+\alpha^{-1}(i, j+N) S^{m}\left(\tau, \mu_{j}\right)\right\rangle d \tau\right. \\
& +\alpha(k, i+N) \int_{0}^{x} e^{d(i+N)(x-\tau)}\left\langle\alpha^{-1}(i+N, j) F^{m}\left(\tau, \mu_{j}\right)\right. \\
& \left.\left.\left.+\alpha^{-1}(i+N, j+N) S^{m}\left(\tau, \mu_{j}\right)\right\rangle d \tau\right]\right\}
\end{aligned}
$$

e

$$
\begin{aligned}
& v^{m}\left(x, \mu_{k}\right)=\sum_{i=1}^{N}\left\{\alpha(k+N, i) e^{d(i)(x-H)} \Gamma_{i}^{m}+\alpha(k+N, i+N) e^{d(i+N) x} \Gamma_{i+N}^{m}\right. \\
& +\sum_{j=1}^{N}\left[\alpha(k+N, i) \int_{H}^{x} e^{d(i)(x-\tau)}\left\langle\alpha^{-1}(i, j) F^{m}\left(\tau, \mu_{j}\right)+\alpha^{-1}(i, j+N) S^{m}\left(\tau, \mu_{j}\right)\right\rangle d \tau\right. \\
& +\alpha(k+N, i+N) \int_{0}^{x} e^{d(i+N)(x-\tau)}\left\langle\alpha^{-1}(i+N, j) F^{m}\left(\tau, \mu_{j}\right)\right. \\
& \left.\left.\left.+\alpha^{-1}(i+N, j+N) S^{m}\left(\tau, \mu_{j}\right)\right\rangle d \tau\right]\right\}
\end{aligned}
$$

onde $d(i), \alpha(i, j)$ e $\alpha^{-1}(i, j)$ denotam, respectivamente, os autovalores da matriz $A$ e os coefecientes das matrizes $X$ e $X^{-1}$. Determinadas as incógnitas $\Gamma_{i}$ pela aplicação das condições de contorno (3.3-3.4), a solução para $\Psi^{m}\left(x, \mu_{k}\right)$ é dada pela equação (4.1), onde $u^{m}\left(x, \mu_{k}\right)$ e $v^{m}\left(x, \mu_{k}\right)$ são expressos nas equações (4.9-4.10).

\section{Resultados Numéricos e Conclusões}

Para testar a eficiência da metodologia proposta, sob o ponto de vista da modelagem computacional, considera-se um domínio homogêneo de $50 \mathrm{~cm}$ de espessura, com condições de contorno: reflexiva à esquerda e vácuo à direita. A seção de choque macroscópica total é $1.0 \mathrm{~cm}^{-1}$, enquanto que para a seção de choque macroscópica 
de espalhamento tomam-se os valores $0.999,0.9,0.8$ e $0.5 \mathrm{~cm}^{-1}$. Assume-se que a velocidade dos nêutrons é $10^{6} \mathrm{~cm} / \mathrm{s}$. Esse domínio unidimensional possui uma fonte de nêutrons unitária na região $0<x<10$. Subitamente, em $t=0$, a fonte de nêutrons é removida. Esse problema é descrito matematicamente pelas equações (2.1-2.4), sendo a condição inicial $\Phi(x, \mu)$ solução do problema

$$
\begin{gathered}
\mu \frac{\partial}{\partial x} \Phi(x, \mu)+\sigma_{t} \Phi(x, \mu)=\frac{\sigma_{s}}{2} \int_{-1}^{+1} \Phi(x, \mu) d \mu+Q(x) \\
\Phi(0, \mu)=\Phi(0,-\mu), \mu>0
\end{gathered}
$$

e

$$
\Phi(H, \mu)=0, \mu<0
$$

onde

$$
Q(x)=\left\{\begin{array}{lll}
1, & \text { se } & 0<x<10 \\
0, & \text { se } & 10<x<50
\end{array} .\right.
$$

Nas tabelas são apresentados valores para taxa de absorção, na região $0<x<$ 45, que é definida pela expressão

$$
T_{a}(t)=\sigma_{a} \int_{-1}^{+1} \int_{45}^{50} \Psi(x, \mu, t) d x d \mu .
$$

Tabela 1. Resultados numéricos pelos métodos $\mathrm{LTS}_{2 \mathrm{~N}}$ e $\mathrm{LTA}_{\mathrm{N}}$, para a taxa de absorção, com $L=500$.

\begin{tabular}{||r||r||r||}
\hline$\sigma_{s}$ & $L T S_{2}$ & $L T A_{1}$ \\
\hline 0,999 & $1.226996 \mathrm{E}-001$ & $1.066211 \mathrm{E}-001$ \\
\hline 0,9 & $8.014036 \mathrm{E}-009$ & $3.721099 \mathrm{E}-010$ \\
\hline 0,8 & $2.192492 \mathrm{E}-012$ & $2.919280 \mathrm{E}-014$ \\
\hline 0,5 & $2.092488 \mathrm{E}-019$ & $2.414999 \mathrm{E}-022$ \\
\hline$\sigma_{s}$ & $L T S_{4}$ & $L T A_{2}$ \\
\hline 0,999 & $1.260764 \mathrm{E}-001$ & $1.268088 \mathrm{E}-001$ \\
\hline 0,9 & $1.644483 \mathrm{E}-008$ & $1.535277 \mathrm{E}-008$ \\
\hline 0,8 & $1.746796 \mathrm{E}-011$ & $1.346287 \mathrm{E}-011$ \\
\hline 0,5 & $5.746516 \mathrm{E}-016$ & $1.494190 \mathrm{E}-016$ \\
\hline *Lê-se como $1.226996 \times 10^{-1}$
\end{tabular}

Para determinar o fluxo angular, inicialmente discretiza-se a variável temporal usando diferenças finitas atrasadas, com $\Delta t=10^{-6} s$, sendo que a solução do conjunto de problemas dado pelas equações (3.2-3.4) é obtida através do método LTA $_{N}$ com $N=1$ e 2. As integrais de convolução que aparecem nas equações (4.9-4.10) são resolvidas numericamente, devido a característica recursiva dos termos de fonte 
$F^{m}\left(x, \mu_{n}\right)$ e $S^{m}\left(x, \mu_{n}\right)$. Para tanto, o domínio espacial foi subdividido em intervalos $\left[x_{i}, x_{i+1}\right]$, com $i=0,1, \ldots, L$, e as funções $u^{m}\left(x, \mu_{n}\right)$ e $v^{m}=\left(x, \mu_{n}\right)$ são interpoladas por splines cúbicos em cada um destes subintervalos.

Na tabela 1, apresentam-se os valores da taxa de absorção na região $45<x<50$, no instante $t=10^{-5}$, obtidos pelos métodos $\mathrm{LTS}_{2 \mathrm{~N}}$ e $\mathrm{LTA}_{\mathrm{N}}, L=500, N=1$ e 2 , e $\sigma_{s}$ assumindo os valores $0.999,0.9,0.8$ e $0.5 \mathrm{~cm}^{-1}$. Os resultados apresentaram uma boa coincidência, levando-se em conta a equivalência entre as aproximações $A_{N}$ e $\mathrm{S}_{2 \mathrm{~N}}$ [3]. Deve-se ressaltar também que o método $\mathrm{LTA}_{\mathrm{N}}$ apresenta uma aparente maior taxa de convergência, pois calculando-se a taxa de absorção pelo método $\operatorname{LTS}_{12}$, considerando $t=10^{-5}, L=500$ e $\sigma_{s}=0.999 \mathrm{~cm}^{-1}$, obtém-se o valor $1.266514 \times 10^{-1}[10]$.

Tabela 2. Resultados numéricos para a taxa de absorção, obtidos pelos métodos $\mathrm{LTS}_{4}$ e LTA 2 , considerando $\sigma_{s}=0.999 \mathrm{~cm}^{-1}, t=10^{-5} s$.

\begin{tabular}{||c||r||r||}
\hline$L$ & $L_{T}$ & $L T S_{4}$ \\
\hline 440 & $1.261702 \mathrm{E}-001$ & $1.269027 \mathrm{E}-001$ \\
\hline 450 & $1.261528 \mathrm{E}-001$ & $1.268853 \mathrm{E}-001$ \\
\hline 460 & $1.261362 \mathrm{E}-001$ & $1.268531 \mathrm{E}-001$ \\
\hline 470 & $1.261203 \mathrm{E}-001$ & $1.268528 \mathrm{E}-001$ \\
\hline 480 & $1.261051 \mathrm{E}-001$ & $1.268375 \mathrm{E}-001$ \\
\hline
\end{tabular}

Na tabela 2, são apresentados os valores numéricos obtidos para a taxa de absorção pelos métodos $\mathrm{LTA}_{\mathrm{N}}$ e $\mathrm{LTS}_{2 \mathrm{~N}}$, considerando $t=10^{-5} \mathrm{~s}, N=2, \sigma_{s}=$ $0.999 \mathrm{~cm}^{-1}$, para $L=440,450,460,470$ e 480 . Analisando estes resultados, observase claramente a lenta convergência para a precisão desejada, uma vez que, com $L=480$, ainda não se tem mais do que três dígitos significativos de coincidência. Acredita-se que, resolvendo-se analiticamente as integrais de convolução, o tempo computacional diminuirá e resultados numéricos mais precisos serão então obtidos. Propõe-se voltar as atenções futuras nesta direção. É importante salientar que a formulação do método proposto é simples, podendo facilmente ser estendida a outras classes de problemas, tais como, problemas com espalhamento anisotrópico, problemas de multigrupo de energia, problemas envolvendo multiregiões. Ademais, obteve-se uma solução analítica para cada passo da aproximação em diferenças finitas do fluxo angular.

Baseando-se em resultados obtidos pelo método $\mathrm{LTA}_{N}$ para problemas estacionários de transporte [3], conclui-se que o método $\mathrm{LTA}_{\mathrm{N}}$ somente terá vantagens sobre o método $\mathrm{LTS}_{\mathrm{N}}$ na resolução de problemas, transientes ou estacionários, com altos graus de anisotropia. As dificuldades de programação impostas pelo método $\mathrm{LTA}_{\mathrm{N}}$, em relação ao método $\mathrm{LTS}_{\mathrm{N}}$, não tornam vantajoso o seu uso para problemas com baixos graus de anisotropia. Acredita-se que o objetivo deste trabalho foi alcançado, avaliando as vatangens e desvantagens na utilização do método LTA $_{N}$ em relação ao método $\mathrm{LTS}_{\mathrm{N}}$. 


\title{
Agradecimentos
}

Os autores agradecem à Fundação de Amparo à Pesquisa do Estado do Rio Grande do Sul (FAPERGS) pelo suporte financeiro parcial na elaboração desse trabalho.

\begin{abstract}
In this work, a new approach to solve one-dimensional linear transport problems with time-dependence is proposed, using the combination of the finite difference scheme and the $\mathrm{LTA}_{\mathrm{N}}$ method. For such, the time-derivative of the angular flux is approximated by backward finite differences scheme, transforming the transient transport problem in a set of one-dimensional transport problems which are then recursively solved, one by one, by the application of the LTA $_{N}$ method. This method consist of the solution of the $A_{N}$ equations using the Laplace transform technique at the spatial variable, with analytical inversion. The $A_{N}$ equations are attained separating the angular flux at the positive and negative directions, respectively, as the sum and subtraction of two functions defined only at the positive directions, applying then the idea of the discrete ordinates method. Numerical simulations are presented.
\end{abstract}

\section{Referências}

[1] R.C. Barros, M. Yavuz, M.P. de Abreu, H. Alves Filho, J.A.M. Mello, Progress in spectral nodal methods applied to discrete ordinates transport problems. Progress in Nuclear Energy, 33 (1998), 117-154.

[2] A.V. Cardona, M.T.M.B. Vilhena, Analytical solution for the $\mathrm{A}_{\mathrm{N}}$ approximation, Annals of Nuclear Energy, 24 (1997), 495-505.

[3] A.V. Cardona et al., The one-dimensional $\mathrm{LTA}_{\mathrm{N}}$ solution in a slab with high order of quadrature, in " $18^{\text {th }}$ International Conference on Transport Theory", pp. 260-264, Rio de Janeiro, RJ, 2003.

[4] G. Coppa, P. Ravetto, An Aprroximate method to study the one velocity neutron integral transport equation. Annals of Nuclear Energy, 9 (1982), 169-174.

[5] D. Gottieb, S.A. Orzag, "Numerical Analysis of Spectral Methods: Theory and Applications", SIAM, Philadelphia, 1997.

[6] E.E. Lewis, W.F. Miller Jr., "Computational Methods of Neutron Transport", American Nuclear Society, Illinois, 1983.

[7] G.J. Marchuk, "Methods of Numerical Mathematics", Springer-Verlag, 1975.

[8] J.V.P. de Oliveira, A.V. Cardona, Solução semi-analítica da equação de transporte dependente do tempo. em "Seleta do XXII CNMAC" (J.M. Balthazar, S.M. Gomes e A. Sri Ranga, eds.), TEMA - Tendências em Matemática Aplicada e Computacional, Vol. 1, No. 1, pp. 193-200, SBMAC, 2000.

[9] J.V.P. de Oliveira et al., Solução semi-analítica da equação de transferência radiativa não-linear, in " $8^{\text {th }}$ Brazilian Congress of Thermal Engineerin and Sciences", ISBN 85-85769-04-01, Porto Alegre, 2000. 
[10] J.V.P. de Oliveira, A.V. Cardona, M.T. Vilhena, Solution of the onedimensional time-dependent discrete ordinates problem in a slab by the spectral and LTS $_{\mathrm{N}}$ methods. Annals of Nuclear Energy. 29 (2002), 13-20.

[11] J.V.P. de Oliveira et al., A semi-anlytical numerical method for time-dependent radiative transfer problems in slab geometry with coherent isotropic scattering. Journal of Quantitative Spectrospopy 83 Radiative Transfer, 73 (2002), 55-66.

[12] J.V.P de Oliveira, A.V. Cardona, Solução da Equação Transiente de Transporte em Domínio Semi-infinito pela Combinação dos Métodos Espectral e $\mathrm{LTS}_{\mathrm{N}}$, em "Seleta do XXIV CNMAC" (E.X.L. de Andrade, J.M. Balthazar, S.M. Gomes, G.N. Silva, A. Sri Ranga, eds.), TEMA - Tendências em Matemática Aplicada e Computacional, Vol. 3, No. 1, pp. 157-164, SBMAC, 2002.

[13] J.V.P. de Oliveira, A.V. Cardona, M.T. Vilhena. Solução da equação transiente de transporte pela combinação dos métodos de diferenças finitas e LTS $_{\mathrm{N}}$ ", Anais em CD do "XII Encontro Nacional de Física de Reatores e Termohidráulica" (XII ENFIR), Rio de Janeiro, RJ, 2000.

[14] C.F. Segatto, M.T. Vilhena, The state-of-the-art of the $L T S_{N}$ method. in International Conference in Reactor Physics and Environmental Analysis in Nuclear Applications, Madrid, 2 (1999), 1618-1631. 